\title{
Sprach- und Weltalternativen: Mehrsprachigkeit als Ideologiekritik in kontrafaktischen Werken von Quentin Tarantino und Christian Kracht
}

\author{
MICHAEL NAVRATIL
}

\begin{abstract}
Alternatives of Languages and Worlds: Multilingualism as Critique of Ideology in Contrafactual Fiction by Quentin Tarantino and Christian Kracht. Multilingualism and the alternate history genre have something in common: both phenomena are based on the construction of alternatives, in the case of multilingualism on the alternatives between different languages and communication systems, and in the case of the alternate history genre on the alternatives between real-world facts and the variation thereof within fictional worlds. This article investigates the interconnections between these two forms of thinking in alternatives by looking specifically at Quentin Tarantino's counterfactual war film Inglourious Basterds (2009) and Christian Kracht's alternate history novel Ich werde hier sein im Sonnenschein und im Schatten (2008). I argue that the consideration of language alternatives forms part of the meta-reflection of the alternate history genre in these works while at the same time opening up a political perspective: in Tarantino's film and Kracht's novel, multilingualism serves as a means for the critique of ideology by rendering palpable the political threats of a worldview based on clear-cut alternatives. In the article's final section, I plead for the establishment of stronger links between the research on literary multilingualism and the theory of fiction.
\end{abstract}

Keywords: multilingualism; counterfactual fiction; alternate history; political writing; theory of fiction; German studies; film studies; Quentin Tarantino; Christian Kracht

\section{Alternativen: Sprachen, Welten und das Politische}

Kontrafaktische Spekulationen haben in der Geschichtswissenschaft einen schlechten Ruf. Obwohl seit den 1980er Jahren immer wieder Versuche einer Verteidigung des kontrafaktischen Denkens unternommen wurden, steht die Mehrzahl der Fachvertreter Überlegungen des Typs „Was wäre gewesen, wenn Napoleon die Schlacht von Waterloo gewonnen hätte" nach wie vor kritisch gegenüber (vgl. Neitzel 2003: 312). Die Einwände betreffen dabei nicht nur 
methodische Probleme des kontrafaktischen Denkens, etwa die Schwierigkeit, plausible Alternativen zum realen Geschichtsverlauf zu modellieren. Kontrafaktische Szenarien haben im Lager der Geschichtswissenschaft auch mehrfach ethisch-politische Bedenken hervorgerufen. So konstatiert etwa Richard J. Evans, der derzeit wohl prominenteste Kritiker kontrafaktischen Denkens: „Tatsächlich gibt es, wenn überhaupt, kaum kontrafaktische Szenarien, die aus einer linken Perspektive verfasst sind. [...] In der Praxis sind kontrafaktische Szenarien [...] mehr oder weniger ein Monopol der Konservativen." (Evans 2014: 62 f.) Evans plausibilisiert diese These dabei unter anderem mit der Beobachtung, dass sich historische kontrafaktische Spekulationen „fast ausschließlich mit traditioneller, altmodischer Politik-, Militär- und Diplomatiegeschichte von der Art [beschäftigten], wie sie in den 1950er Jahren vorherrschte." (Evans 2014: 171) Betont würden also vor allem die Biografien und Taten ,großer Männer, Kriege, Schlachten und Attentate. Die reale Komplexität historischer, sozialer und politischer Determinationsprozesse, deren Berücksichtigung von einer politisch eher links orientierten oder postmodernen Geschichtstheorie angemahnt wird, trete somit in der kontrafaktischen Historiografie zurück hinter die Imagination simpler, monokausaler Alternativen des Geschichtsverlaufs.

Blickt man nun auf das künstlerisch-fiktionale Parallelphänomen zum kontrafaktischen Denken in der Geschichte, nämlich auf Romane und Filme des Alternate History-Genres wie Philip K. Dicks The Man in the High Castle oder Christoph Ransmayrs Morbus Kitahara, so lassen sich zunächst einige Gemeinsamkeiten erkennen: Werke der fiktionalen Kontrafaktik ${ }^{1}$ basieren ebenfalls auf einer eindeutigen Alternativbildung zwischen Fakten der realen Welt und ihrer künstlerischen Variation innerhalb einer fiktionalen Welt. Darüber hinaus zeigt auch die Kontrafaktik in Literatur und fiktionalem Film eine starke Affinität zum Politischen (vgl. Navratil 2019). Eine Verbindung von kontrafaktischem und konservativ-reaktionärem Denken jedoch, wie sie

Mit dem Konzept der Kontrafaktik werden die Bedingungen und Funktionen des kontrafaktischen Erzählens speziell in fiktional-künstlerischen Medien fokussiert. Ich habe folgende Definition vorgeschlagen: „Kontrafaktik liegt vor, wenn in einem fiktionalen Medium realweltliches Faktenmaterial auf signifikante Weise variiert wird." (Navratil 2019: 366) Derartige Faktenvariationen der Kontrafaktik finden sich nicht nur in alternativgeschichtlichen Romanen und Filmen, sondern auch in utopischen und dystopischen Texten, in Doku- und Autofiktionen, Satiren oder im Schlüsselroman. Eine ausführliche fiktionstheoretische Fundierung und Funktionserläuterung der Kontrafaktik findet sich in meiner Dissertationsschrift: Kontrafaktik der Gegenwart. Politisches Schreiben als Realitätsvariation bei Christian Kracht, Kathrin Röggla, Juli Zeh und Leif Randt (Navratil, in Vorbereitung). 
NAVRATIL

innerhalb der Geschichtswissenschaft mitunter moniert wurde, wird man mit Blick auf das literarische oder filmische Alternate History-Genre kaum plausibel finden können. Zwar ist die politische Problematik eines simplen Alternativendenkens auch im fiktionalen Bereich nicht einfach ausgesetzt; doch ergeben sich im Rahmen einer „Politik der Literatur“ - also unter der Bedingung einer, wie Jacques Rancière schreibt, „wesentliche[n] Verbindung zwischen der Politik als spezifischer Form kollektiver Praxis und der Literatur als bestimmte[r] Praxis der Kunst des Schreibens" (Rancière 2011: 13) - ganz eigene Möglichkeiten des ästhetischen und reflexiven Umgangs mit dieser Problematik. So nehmen avanciert-postmoderne Beispiele des Alternate History-Genres mitunter gerade das eigene, erzählstrukturelle Alternativendenken zum Ausgangspunkt, um über die politische Problematik starrer Alternativbildungen nachzudenken.

Im vorliegenden Aufsatz sollen zwei Beispiele einer solchen avanciertpostmodernen, historischen Kontrafaktik in den Blick genommen werden: Quentin Tarantinos kontrafaktischer Kriegsfilm Inglourious Basterds von 2009 und Christian Krachts Alternate History-Roman Ich werde hier sein im Sonnenschein und im Schatten von 2008. ${ }^{2}$ Es soll gezeigt werden, dass das Konzept der ,Alternative' für die genannten Werke nicht nur qua Genre von Bedeutung ist, sondern auch binnenfiktional thematisch wird. Die Verhandlung des Alternativendenkens trägt in Tarantinos Film und Krachts Roman zur Genrereflexion bei, erfüllt darüber hinaus aber auch eine politischkritische Funktion.

Der Fokus der folgenden Ausführungen liegt speziell auf dem Einsatz der literarischen - respektive allgemein künstlerischen - Mehrsprachigkeit in den genannten Werken. Eine Verbindung von Kontrafaktik und Mehrsprachigkeit liegt insofern nahe, als sich beide Phänomene als Formen des Alternativendenkens begreifen lassen: im Falle der Mehrsprachigkeit der Alternativen zwischen verschiedenen Sprachen oder Sprachformen, im Falle der Kontrafaktik zwischen Fakten der realen Welt und deren Variation innerhalb einer fiktionalen Welt. Nun trägt literarische Mehrsprachigkeit keineswegs zwingend dazu bei, bestehende politische, nationale oder identitäre Oppositionen und Alternativen zu zementieren oder zu naturalisieren: Im Gegenteil steht der Einsatz literarischer Mehrsprachigkeit in der Moderne und Postmoderne für gewöhnlich im Zeichen eines „vertieften Bewußtseins von der Kontingenz und Umgestaltbarkeit sprachlicher und anderer Zeichen- und

\footnotetext{
Auch Kathleen Singles diskutiert die genannten Werke Krachts und Tarantinos als Beispiele eines ,hyper-selbstreflexiven' Umgangs mit dem Genre der Alternate History. Vgl. Singles (2013: 247-278).
} 
Regelsysteme“ (Schmitz-Emans 2004: 16), einer erhöhten Sprachreflexivität (vgl. Radaelli 2011: 38) sowie eines ethisch motivierten „Interesse[s] für eine Hermeneutik der Differenz und der Fremdheit" (Sepp 2020: 53). Genau diese Eigenschaften ermöglichen es der Mehrsprachigkeit in den kontrafaktischen Werken Krachts und Tarantinos, auf die politische Problematik eines starren Alternativendenkens hinzuweisen und damit zugleich auch jene harten Oppositionen, die dem Genre der Alternate History erzählstrukturell zugrunde liegen, binnenfiktional zu unterlaufen.

\section{Quentin Tarantino: Inglourious Basterds}

Quentin Tarantinos Film Inglourious Basterds verfolgt eine Reihe unterschiedlicher Erzählstränge in den Jahren zwischen 1941 und 1944. Im Zentrum der Handlung steht eine fiktive, jüdisch-amerikanische Guerilla-Truppe namens ,Inglourious Basterds' unter der Führung von Lieutenant Aldo Raine (gespielt von Brad Pitt), die im besetzten Frankreich Jagd auf Nazis macht, sowie SSStandartenführer Hans Landa (gespielt von Christoph Waltz), genannt ,the Jew Hunter, der die Inglourious Basterds ausfindig zu machen sucht. Während der Premiere eines von Joseph Goebbels gedrehten Kriegsfilms in einem französischen Kino, bei der auch Adolf Hitler zugegen ist, lassen sich einige Mitglieder der Basterds ins Kino einschmuggeln und erschießen Hitler, Goebbels und einen großen Teil der versammelten Nazi-Prominenz, wobei sie bei ihrem Anschlag durch eine jüdische Holocaust-Überlebende sowie Hans Landa unterstützt werden.

Der Vielzahl an politischen, nationalen und ideologischen Parteien korrespondiert im Film eine Vielzahl an Sprachen: Inglourious Basterds ist ein viersprachiger Film (deutsch, englisch, französisch, italienisch), wobei die einzelnen Figuren des Films jeweils unterschiedlich - und mitunter auch gar nicht - polyglott sind. Politisch semantisiert wird dabei nicht so sehr die einzelne Sprache, sondern vielmehr die Pragmatik des strategischen Sprachwechsels: Mehrsprachigkeit fungiert in Inglourious Basterds vor allem als Mittel der Manipulation und politischen Gewaltausübung.

Die Möglichkeit eines Missbrauchs von Mehrsprachigkeitskompetenz kommt bereits in der Eingangsszene des Films eindrücklich zur Darstellung: SS-Standartenführer Hans Landa trifft während der deutschen Besatzung Frankreichs mit seinen Soldaten auf dem Hof des Landwirts Perrier LaPadite (gespielt von Denis Ménochet) ein. In fließendem Französisch wendet sich Landa an LaPadite und lässt sich in dessen Haus einladen. Dort teilt er dem Landwirt jedoch mit, dass sein Französisch zu schlecht sei, um eine Konversation sicher manövrieren zu können, und bittet LaPadite, ins Englische 
NAVRATIL

wechseln zu dürfen - welches er, Landa, sodann mit ähnlicher Geläufigkeit spricht wie zuvor das Französische. Im Fortgang des Gesprächs, welches zunächst ohne erkennbare Spannungen verläuft, kann Landa LaPadite dann zu dem Geständnis bewegen, dass letzterer eine jüdische Familie in seinem Haus versteckt hält.

Die folgende Passage aus dem Drehbuch gibt den Wendepunkt des Gesprächs sowie den damit verbundenen Sprachwechsel wieder. In eckigen Klammern ist der im Film tatsächlich zu hörende französische Text notiert:

The farmer, pipe in mouth, stares across the table at his German opponent.

COL. LANDA: You are sheltering enemies of the state, are you not?

PERRIER: Yes.

COL. LANDA: You're sheltering them underneath your floorboards, aren't you?

PERRIER: Yes.

COL. LANDA: Point out to me the areas where they're hiding.

The farmer points out the areas on the floor where the Dreyfuses are underneath.

COL. LANDA: Since I haven't heard any disturbance, I assume that while they're listening, they don't speak English?

PERRIER: Yes.

COL. LANDA: I'm going to switch back to French now, and I want you to follow my masquerade - is that clear?

PERRIER: Yes.

Col. Landa stands up from the table and, switching to FRENCH, says, SUBTITLED IN ENGLISH:

COL. LANDA: [Monsieur LaPadite, je vous remercie pour le lait et votre hospitalité. Il me semble que nous en avons terminé.] Monsieur LaPadite, I thank you for your milk and your hospitality. I do believe our business here is done.

The Nazi officer opens the door and silently motions for his men to approach the house.

COL. LANDA: [Ah, mesdames, je vous remercie pour le temps que vous m'avez consacré. Nous n'ennuierons pas votre famille plus longtemps.] Madame LaPadite, I thank you for your time. We shan't be bothering your family any longer.

The soldiers enter the doorway. Col. Landa silently points out the areas of the floor the Jews are hiding under.

COL. LANDA: [Donc, Monsieur, Mesdemoiselles, je prends congé de vous et je vous dis adieu.] So, Monsieur and Madame LaPadite, I bid you adieu.

He motions to the soldiers with his index finger.

They TEAR UP the wooden floor with MACHINE-GUN FIRE. (Tarantino 2009: 15 f.; Tarantino [Regie] 2009) 
Mehrsprachigkeit erweist sich hier letztlich als Werkzeug zur umso effizienteren Menschenjagd: Da die Familie Dreyfus der englischen Konversation nicht zu folgen vermag, ist sie im Anschluss dem Angriff durch die NaziSoldaten schutzlos ausgeliefert. Der initiale Eindruck Hans Landas als charmantem, polyglottem Weltbürger und freundlichem Gesprächspartner wird damit Lügen gestraft. Ein Nazi, so ließe sich die politische Aussage der Szene etwas lapidar zusammenfassen, bleibt eben auch dann ein Nazi, wenn er elegant Französisch parliert.

Auch im weiteren Verlauf von Inglourious Basterds wird Mehrsprachigkeit wiederholt in Verbindung gebracht mit den Versuchen einer strategischen Identitätsbemäntelung. In immer neuen Varianten entwirft der Film Situationen, in denen die Figuren ihr Gegenüber dadurch zu täuschen suchen, dass sie eine Sprache sprechen, die nicht die eigene ist (wobei diese Versuche bezeichnenderweise häufig scheitern). ${ }^{3}$ Die Opposition zwischen verschiedenen Sprachen steht bei Tarantino allerdings in keiner klaren Parallele zur Opposition der Kriegsparteien. Die simple Alternative etwa von (bösen) Nazis und (guten) Amerikanern, wie sie für den amerikanischen Film über den Zweiten Weltkrieg geradezu topisch ist, wird in Inglourious Basterds beständig unterlaufen (vgl. Butter 2015). In bewusster Vermeidung nationalistischer Metonymien verläuft die politische Trennlinie in Inglourious Basterds nicht zwischen bestimmten Sprachen und Nationen, sondern vielmehr im politischen Bereich selbst, also im Feld der politischen Ideologien und vor allem der konkreten politischen Handlungen, die im Film häufig Gewalthandlungen sind. Inglourious Basterds redet mithin keinem sprachlichen Essenzialismus oder gar Nationalismus das Wort; strategische Versuche der Selbstkonstruktion qua Sprachwechsel werden lediglich da problematisiert, wo sie substanziellere Oppositionen im politischen Bereich bemänteln sollen. (Im obigen Beispiel etwa sind ja sowohl Landa als auch LaPadite zum Sprachwechsel befähigt; nur setzt letzterer seine Mehrsprachigkeitskompetenz eben nicht zur Verfolgung Unschuldiger ein, sondern im Gegenteil, um die jüdische Familie zu schützen und wird entsprechend als positive Figur aufgebaut.) Der Film macht somit deutlich, dass politische Handlungen sich zwar sprachlich verleugnen lassen,

3 Anders als in Tarantinos früherem Filmschaffen lässt sich in Inglourious Basterds wohl aufgrund des historischen Themas - ein verhaltener identitärer Essenzialismus beobachten, insbesondere in Bezug auf Fragen der ethnischen Zugehörigkeit: „Whereas True Romance, Reservoir Dogs, and Pulp Fiction had (to varying degrees) suggested that individuals could transcend ethnic and racial barriers by enacting effective performances, Inglourious Basterds contends that one's actual ethnic background greatly aids or restricts his or her ability to perform different identities." (Cavallero 2011: 148). 
NAVRATIL

durch bloße sprachliche Gegenbehauptung aber nicht ungeschehen gemacht werden können.

Die Fähigkeit, Sprachen beliebig zu wechseln, wird am Ende des Films enggeführt mit dem Versuch, die Geschichte selbst umzuschreiben. Als der ,Jew Hunter Landa erkennen muss, dass ein Sieg der Deutschen zusehends unwahrscheinlich wird und er somit Gefahr läuft, sich nach dem verlorenen Krieg vor einem jüdischen Tribunal für seine Taten verantworten zu müssen, schließt er einen Deal mit den Basterds und der englischen Regierung: Im Gegenzug für eine Beteiligung am Hitler-Attentat sollen Landas Verbrechen an den Juden öffentlich als notwendige Tarnaktionen ausgegeben und er selbst in den Status eines finanziell wohlversorgten Kriegshelden erhoben werden. Die Option einer für Landa opportunen Geschichtslüge wird dabei in Verbindung gebracht mit seiner Fähigkeit, sich mühelos durch unterschiedliche Sprachsysteme zu bewegen: Schließlich würde die Umdeutung von Landas Verbrechen als Heldentaten das Meisterstück unter seinen vielfältigen Verstellungsversuchen darstellen; auch bildet Landas perfekte Beherrschung des Englischen die Bedingung, um allererst mit der englischen Regierung sowie mit den sprachlich weitgehend inkompetenten Basterds verhandeln zu können.

Das Thema der Geschichtsumschreibung stellt darüber hinaus eine metareflexive Verbindung zum Alternate History-Genre her: Tarantinos Film, der als Werk der Kontrafaktik strukturell auf einer Variation der Realgeschichte beruht, wirft innerhalb der kontrafaktischen Diegese - also gleichsam metafaktisch ${ }^{4}$ - die Frage auf, ob eine Geschichtslüge, welche sich metonymisch auf den totalen Signifikanten Auschwitz verlängern lässt, jemals gerechtfertigt sein kann. Die Antwort auf diese Frage fällt im Film entschieden negativ aus: Nachdem Hitler und die Nazi-Eliten dem Attentat zum Opfer gefallen sind, schneidet Aldo, der Anführer der Basterds, dem ,Jew Hunter Landa mit einem gewaltigen Fleischermesser ein Hakenkreuz in die Stirn. Die Wahrheit über Landas Verbrechen wird somit dem Körper selbst in Form eines politischen Kainsmals eingeschrieben, sodass die Option der bloß sprachlichen Gegenbehauptung hinfällig wird.

Letzten Endes besteht der Film auf der unbedingten Gültigkeit und politischen Relevanz historischer Wahrheit, und zwar unter bewusster Ausnutzung des (scheinbaren) Paradoxes, dass es sich bei Inglourious Basterds

4 Mit dem Begriff Metafaktizität (engl. metafactuality) bezeichne ich Reflexionen über Wahrheit, Lüge oder Fälschung innerhalb der fiktionalen Welten der Kontrafaktik (vgl. Navratil, im Druck). 
Sprach- und Weltalternativen

um ein Werk der Kontrafaktik handelt. ${ }^{5}$ So wie Mehrsprachigkeit im Film auf der Handlungsebene als potenzielles Mittel eines problematischen Machthandelns desavouiert wird, der Film selbst - als mehrsprachiges Kunstwerk betrachtet - aber zugleich die Pluralität der Sprachen sowie die künstlerischen Einsatzmöglichkeiten der Mehrsprachigkeit zelebriert, so nutzt auch Inglourious Basterds die Möglichkeiten einer Etablierung kontrafaktischer Weltalternativen im Genre der Alternate History ausgerechnet dazu, um sich auf der Handlungsebene entschieden gegen die Option der Geschichtsklitterung zu verwahren.

\section{Christian Kracht: Ich werde hier sein im Sonnenschein und im Schatten}

Drittes Reich, Zweiter Weltkrieg und Holocaust bildeten in der zweiten Hälfte des 20. Jahrhunderts zweifellos die dominierenden Themen des Alternate History-Genres (vgl. Rosenfeld 2005). Etwa seit Ende des Kalten Krieges finden sich nun wieder vermehrt Werke des Alternate History-Genres, die auch auf andere historische Stoffe zurückgreifen. Eines der prominentesten Beispiele aus dem deutschsprachigen Raum ist Christian Krachts 2008 veröffentlichter Roman Ich werde hier sein im Sonnenschein und im Schatten. ${ }^{6}$ In dessen fiktionaler Welt befindet sich die kontrafaktisch von Lenin gegründete Schweizer Sowjet-Republik - die SSR - bereits seit fast einhundert Jahren im Krieg, unter anderem mit dem faschistischen Deutschland im Norden. Der namenlose Protagonist des Romans, ein schwarzer, ursprünglich aus Afrika stammender Militär der SSR, reist durch die winterlichen Landschaften der Schweiz und gelangt schließlich zum Réduit, dem gigantischen Tunnelsystem in den Schweizer Alpen. Nachdem er allerdings die umfassende Dekadenz im untertunnelten Herzen der Schweiz erkannt hat, kehrt er als Messias mit leuchtend blauen Augen nach Afrika zurück und führt die Menschen aus den Kolonialstädten zurück in die Savanne.

5 Ungeachtet ihrer fiktiven Aussagegegenstände lassen sich (Geschichts-)Lügen und Kontrafaktik auf einer pragmatischen Ebene eindeutig voneinander unterscheiden: Während Lügen Täuschungsabsicht verfolgen, operieren kontrafaktische Aussagen mit Annahmen, welche „sowohl für diejenigen, die sie vortragen, als auch für diejenigen, an die sie sich richten, offensichtlich falsch sind." (Danneberg 2009: 287) Siehe zum Verhältnis von Lüge und Kontrafaktik auch Navratil (im Druck).

6 Die folgenden Überlegungen bilden einen Auszug aus der umfänglicheren Analyse von Krachts Roman in meiner Dissertationsschrift (Navratil, in Vorbereitung). 
NAVRATIL

Im Zentrum von Ich werde hier sein... steht die Frage, ob Alternativen zu den Ideologien der Moderne möglich sind. Bei der künstlerischen Auseinandersetzung mit dieser Frage wird auch das Verhältnis von Sprache, Realität und politischer Gewalt verschiedentlich durchdekliniert (vgl. Nover 2012: 177-286). So erinnert sich der aus Afrika stammende Protagonist folgendermaßen an seine militärische Ausbildung:

Nach einer Weile sprachen wir auch untereinander kein Chiwa mehr, sondern Schweizer Mundart. Wir hörten die in Wachs eingebrannten Stimm-Schriften von Karl Marx und die Geschichte des grossen Eidgenossen Lenin, der, anstatt in einem plombierten Zug in das zerfallene, verstrahlte Russland zurückzukehren, in der Schweiz geblieben war, um dort nach Jahrzehnten des Krieges den Sowjet zu gründen [...]. (Kracht 2008: 57 f.)

Der Übergang vom „Chiwa“ zum Schweizerdeutsch bildet für den afrikanischen Soldaten die Grundvoraussetzung für das Vertrautwerden mit der europäischen Geschichte sowie für den Eintritt in eine militaristische Weltanschauung. Thematisiert wird im zitierten Textausschnitt darüber hinaus die mediale Codierbarkeit von Sprache, welche für den gesamten Roman von großer Bedeutung ist. In Krachts Roman ist die Schweiz auf eine Kulturstufte der Schriftlosigkeit regrediert, wobei das Verlernen der schriftlichen Kommunikation einen „Prozess des absichtlichen Vergessens“ (Kracht 2008: 43) bildet, welcher die ohnehin dominante Mündlichkeit der Dialekte exklusiv werden lässt: „Unsere Mundarten sind schon immer ausschließlich orale Sprache gewesen, es gab die Niederschrift nur in Hochdeutsch. Die Mundarten sind unser Koiné, der Grund, warum wir nicht Deutsch sprechen." (Kracht 2008: 43) Der ursprünglich aus Afrika stammende Kommissär ist einer der wenigen Schweizer, der die schriftliche Kommunikation noch beherrscht. Seine überlegene Produktivität beweist dabei die Fragwürdigkeit eines dogmatischen Rückzugs auf Dialekt und Oralität.

Neben Mündlichkeit und Schriftlichkeit werden im Verlauf des Romans vom Kommissär noch zwei weitere Sprachformen erprobt: die sogenannte „Rauchsprache“ (erstmals Kracht 2008: 42) sowie die „Morpheme[...] der Erde“ (Kracht 2008: 144). Die Rauchsprache, welche der Protagonist vom Dissidenten Brazhinsky erlernt, ermöglicht es, das Gesprochene gegenständlich werden zu lassen: „[W]ir beginnen, das Gedachte zu sprechen und in den Raum zu stellen. [...] Sprache existiert nicht nur im Raum, sie ist zutiefst dinglich, sie ist ein Noumenon." (Kracht 2008: 44) In der Kritik der reinen Vernunft hatte Kant das ,Noumenon' für einen zwar denkmöglichen, letztlich aber problematischen Begriff erklärt, da dasjenige, was außerhalb der Erfahrungsmöglichkeiten des Menschen liege, eben 
nicht erkannt werden könne (vgl. Kant 1974 [1781]: 279-282). Die Annahme einer strikten Trennung von Erscheinung und ,Ding an sich', die Kant noch transzendentalphilosophisch begründet hatte, wurde in der modernen Semiotik - etwa in der Sprachtheorie Ferdinand de Saussures - sowie im Rahmen des linguistic turn und der Dekonstruktion dann auch sprachphilosophisch produktiv gemacht: Ähnlich wie in der kantschen Epistemologie der direkte Schluss von der bloßen Erscheinung auf das ,Ding an sich unzulässig ist, so erlaubt auch die Sprache als abgeschlossenes semiotisches System keinen unmittelbaren Rückschluss auf das Wesen der Dinge.

In Krachts Roman soll diese Trennung von Erscheinung und Ding an sich nun zurückgenommen werden. Die Möglichkeit einer neuen, unmittelbar dinghaften Sprache wird in Ich werde hier sein... allerdings nur aufgerufen, um schlussendlich wieder verworfen zu werden. Medial betrachtet bildet die Rauchsprache zwar eine Alternative zu den früher erprobten Kommunikationstechniken Mündlichkeit und Schriftlichkeit. In ihrer Funktion als Instrument des Krieges jedoch stimmt sie mit den anderen beiden weitgehend überein: Bereits in der ersten Szene ihres Gebrauchs im Roman dient die Rauchsprache dazu, eine Gruppe von Militärs zu entwaffnen (vgl. Kracht 2008: 108). Auch werden die scheinbar neuen Erkenntnismöglichkeiten der Rauchsprache symbolträchtig konterkariert, wenn sich Brazhinsky letztlich die Augen aussticht, sodass seine vormalige Verblendung - ähnlich wie bei König Ödipus (vgl. Schöll 2014: 301) - am Körper selbst manifest wird. Auf seiner Reise zurück nach Afrika lässt der Protagonist des Romans denn auch die von Brazhinsky erlernte Sprachform hinter sich: „Es war die Sprache der Weissen, ein Idiom des Krieges, und ich brauchte sie nicht." (Kracht 2008: 138)

Nach Mündlichkeit, Schriftlichkeit und Rauchsprache greift der Protagonist am Ende von Ich werde hier sein... noch auf eine vierte Kommunikationsform zurück:

[I]ch legte mit Schilfhalmen meinen Namen in endlosen Bändern auf die staubige Strasse, ich schrieb Wörter, Sätze, ganze Bücher in die Landschaft hinein - die Geschichte der Honigameisen, die Enzyklopädie der Füchse, das Geblüt der Welt [...]. Ich notierte nicht mit Tusche, sondern mit Schrift, mit den Morphemen der Erde. (Kracht 2008: 143)

Mittels dieser neuerlichen utopischen Sprachform, welche die Differenz von Kultur und Natur aufzulösen scheint und damit einen Ausstieg aus den Verwicklungen menschlicher Kultur und menschlicher Grausamkeit verspricht, wird hier auf den utopischen Schluss des Romans vorausgedeutet, wenn die Menschen die modernen Städte verlassen und in der Savanne Afrikas verschwinden. Es stellt sich allerdings die Frage, ob die Unmöglichkeit einer 
NAVRATIL

radikalen Alternative, welche anhand der vorangegangenen Sprachformen vorgeführt wurde, nicht auch noch die Natursprache betrifft. So bildet die Alternative von „Tusche“ und „Schrift“ keine semantisch plausible Opposition; und Ausdrücke wie „Geschichte der Honigameisen“ oder „Enzyklopädie der Füchse“ scheinen bereits rein lexikalisch tief durchtränkt von überkommenen kulturellen Vorstellungen.

Tatsächlich verweist der Schluss des Romans denn auch auf die Unmöglichkeit eines forcierten Austritts aus der Moderne. Der letzte Satz des Protagonisten im Roman lautet: „Und die blauen Augen unserer Revolution brannten mit der notwendigen Grausamkeit." (Kracht 2008: 147) Bei diesem Satz handelt es sich um eine Übersetzung zweier Verse aus Louis Aragons Front Rouge: „Les yeux bleus de la Révolution / brillent d'une cruauté nécessaire“ (Aragon 1931: 16). Der Aufbruch in eine vermeintlich neue Natürlichkeit wird hier mit den Worten eines surrealistischen - und mithin genuin modernen Dichters charakterisiert. Die Verkündigung des Austritts aus Zivilisation und kommunistischer Ideologie erweist sich somit selbst als intertextuelles Zitat, welches darüber hinaus ausgerechnet auf einen Text referiert, mit welchem der Autor Aragon den Stalinismus zu rechtfertigen suchte (vgl. Lorenz 2018: 435). Aber auch, wenn man den intertextuellen Verweis beiseiteließe, würde das letzte Wort, das der messianische Protagonist im Buch spricht, immer noch „Grausamkeit“ lauten. In der Rechtfertigung dieser Grausamkeit hallen just jene Legitimationsversuche für die Kriege der SSR nach, die der Protagonist doch eigentlich zu überwinden getrachtet hatte. Der Ausbruch aus der Moderne scheint somit letztlich in die Antinomien der Moderne zurückzuführen: in Weltanschauung, Krieg und Unterdrückung. Entsprechend werden auch die unterschiedlichen Sprachen und Sprachsysteme in Ich werde hier sein... nicht angeführt, um eine Hierarchisierung zwischen ihnen vorzunehmen. Krachts Roman deutet vielmehr darauf hin, dass der Versuch, die eine, einzig wahre Sprache oder Sprachform jenseits der Ideologien zu finden, selbst immer schon ideologisch ist.

Konsequenterweise korrespondiert das Scheitern der sprachlichen und der politischen Alternativen in Krachts Roman mit der Dekonstruktion des Alternate History-Genres selbst. Anders als konventionellere Beispiele des Genres kontrastiert Ich werde hier sein... nicht mehr die reale Geschichte mit einem normativ deutlich schlechteren oder deutlich besseren alternativen Geschichtsverlaufs. Stattdessen macht der Roman darauf aufmerksam, dass gerade die Sehnsucht nach klaren Alternativen, wie sie im ästhetischen Bereich unter anderem dem Erzählverfahren der Kontrafaktik selbst zugrunde liegt, den Nährboden für die politischen Katastrophen des 20. Jahrhunderts abgegeben hat. Kracht wägt mithin in seinem Roman keine politischen und 
historischen Alternativen mehr gegeneinander ab, sondern verfasst vielmehr, wie Dietmar Dath es in einer Rezension ausdrückte, „ein Buch gegen die Geschichte als solche." (Dath 2008) Damit bringt Kracht in Ich werde hier sein... das wahrhaft postmoderne Kunststück zustande, eine Alternate HistoryErzählung zu präsentieren, welche die Option klarer politischer, sprachlicher, künstlerischer oder anderweitiger Alternativen ebenso zu Irrtümern und Unmöglichkeiten erklärt wie die Idee des historischen Fortschritts: eine ,Alternativgeschichte' also, aber ohne Alternativen und ohne Geschichte.

\section{Tarantino und Kracht: Ein Vergleich}

Sowohl bei Tarantino als auch bei Kracht wird die Thematisierung sprachlicher Alternativen genutzt, um auf die politische Bedenklichkeit starrer Alternativbildungen hinzuweisen. Tarantinos Inglourious Basterds vermeidet bewusst eine national-sprachessentialistische Bewertung seiner Figuren, weist aber zugleich auf die politischen Grenzen und Bedenklichkeiten rein sprachlicher Konstruktionen hin. Kalkulierte Sprachwechsel werden im Film in Verbindung gebracht mit den Themen der politischen Manipulation und der Geschichtslüge. Ausgerechnet innerhalb eines Werkes der Kontrafaktik wird somit letztlich die unbedingte Relevanz historischer Fakten verteidigt. Krachts Ich werde hier sein im Sonnenschein und im Schatten hingegen exponiert anhand sprachlicher Alternativen die Problematik eines starren Alternativendenkens überhaupt: Ideologie, so führt der Roman vor, besteht nicht darin, in einem ,falschen' politischen Weltbild oder sprachlichen System befangen zu sein; Ideologie besteht vielmehr in der Vorstellung, dass sich die Mehrstimmigkeit menschlichen Lebens in Richtung nur einer Sprach- und Weltalternative überwinden lasse.

Die konkreten Manifestationsformen literarischer Mehrsprachigkeit und ihre politischen Implikationen unterscheiden sich in den genannten Werken allerdings beträchtlich: In Tarantinos Film kommen vier natürliche Sprachen vor. Aufgeworfen werden in diesem Zusammenhang immer wieder die Fragen, wer welche Sprachen wie gut versteht, wie über (fehlende) sprachliche Kompetenzen Ein- und Ausschlüsse produziert werden und bis zu welchem Grade sich Konzepte von einer Sprache in eine andere Sprache übertragen lassen - ein Problem, das auf der Rezeptionsebene nicht zuletzt die Untertitelung des Films betrifft (vgl. Ávila-Cabrera 2013). Der politischkritische Impetus des Films richtet sich dabei weder gegen eine bestimmte Sprachgemeinschaft noch gegen Sprachwechsel und Mehrsprachigkeit als solche (die aufseiten der Zuschauerinnen und Zuschauer ja durchaus als lustvoll erlebt werden). Kritisiert werden lediglich die sprachlichen Verschleierungen 
NAVRATIL

substanzieller politischer Alternativen. Die prinzipielle Möglichkeit gelingender sprachlicher Kommunikation stellt Tarantinos Films dabei ebenso wenig in Frage wie die Unterscheidbarkeit zwischen gutem und schlechtem politischen Handeln oder zwischen Wahrheit und Lüge. Wie so häufig im Rahmen postmoderner Politik (man denke nur an das derzeit so intensiv diskutierte Thema der Fake News) wird auch bei Tarantino der dekonstruktive Impuls nicht unendlich weiterverfolgt, sondern kennt und respektiert gewisse Grenzen: etwa die Materialität des Körpers, politische Grundsätze oder bestimmte historische Wahrheiten, deren willkürliche Umdeutung sich aus ethischen Gründen schlicht verbietet.

In Krachts Ich werde hier sein... wird demgegenüber die Dekonstruktion politischer Ideologien sowie anderer Essenzialismen konsequent zu Ende geführt - um den Preis allerdings, dass der Roman selbst keine positive politische Botschaft mehr zu formulieren vermag. Zwar thematisiert auch Krachts Roman verschiedene natürliche Sprachen, insbesondere Hochdeutsch, Schweizerdeutsch und Chewa; anders als bei Tarantino nimmt diese inhaltlich behauptete Vielzahl der Sprachen jedoch kaum mehr Einfluss auf die manifeste Gestalt des Romantextes (eines der konstitutiven Paradoxa des dominant autodiegetisch erzählten Romans besteht ja gerade darin, dass der Erzähltext des Schweizer Kommissärs auf Hochdeutsch verfasst ist). So wie die inhaltlich behauptete Mehrsprachigkeit auf der Textoberfläche von Ich werde hier sein... weitgehend nivelliert wird, so fallen auch die verschiedenen Sprachformen letztlich funktional in eins: Trotz aller behaupteten Differenz führen sie immer nur wieder zu Krieg und Zerstörung. Im kontinuierlichen Wechsel sowie im konstanten Scheitern der Sprachsysteme wird dabei die generelle Unmöglichkeit symbolisiert, aus den ideologischen Verstrickungen der Moderne auszusteigen, wobei eine zusätzliche, gleichsam antiperformative Volte des Romans darin besteht, dass die utopischen Kommunikationssysteme der Rauch- und Natursprache - in ihrer Eigenschaft als fiktive Kommunikationssysteme - noch nicht einmal vorstellbar sind. Während also in Inglourious Basterds die (politische) Wahrheit letztlich durch alle ästhetischen und sprachlichen Verstellungen hindurchscheint, bildet Mehrsprachigkeit in Ich werde hier sein... nur noch ein Thema unter anderen, anhand derer die Hoffnung auf klare epistemische, sprachliche oder politische Alternativen reflexiv ins Nichts gedreht wird. 


\section{Ausblick: Für eine Fiktionstheorie literarischer Mehrsprachigkeit}

Jenseits einer Verbindung von Mehrsprachigkeit und dem Politischen, wie sie in der philologischen Mehrsprachigkeitsforschung häufig geschlagen wird (vgl. Sepp 2020: 53), wurde im vorliegenden Aufsatz der Versuch unternommen, literarische Mehrsprachigkeit in Beziehung zu setzen mit einem fiktionstheoretisch beschreibbaren Phänomen, nämlich der basalen Referenzstruktur der Kontrafaktik. Derartige Verbindungen von Mehrsprachigkeit mit fiktionsoder erzähltheoretischen Fragestellungen finden sich, wie Rüdiger Zymner bemerkt, in der bisherigen Forschung zur literarischen Mehrsprachigkeit nur selten:

Zum Zusammenhang von Mehrsprachigkeit und Erzählen bzw. zur Mehrsprachigkeit in Erzähltexten gibt es nur wenige wissenschaftliche Arbeiten. Mehrsprachigkeit und Erzählen bzw. Mehrsprachigkeit in Erzähltexten ist bislang kein Thema der Narratologie und steht nicht im Mittelpunkt der literaturwissenschaftlichen Komparatistik. [...] Vielfach interessiert [...] die Thematik der Fremdheit und Fremdheitserfahrungen oder ,des Fremden' in den Erzähltexten mehr als Textstrategien und Verfahren in der narrativen Darstellung von Mehrsprachigkeit. Außerdem ist festzustellen, dass Mehrsprachigkeit in Erzähltexten als textstrukturell-semiotischer Sachverhalt häufig mit der Mehrsprachigkeit der Autorinnen und Autoren als kognitiv-epistemischer Sachverhalt vermischt oder verwechselt wird. (Zymner 2020: 294)

Zymners Befund lässt sich noch einmal verschärfen, wenn man speziell den Bereich der Fiktionstheorie fokussiert (der mit der Narratologie zwar signifikante Überschneidungen aufweist, aber nicht mit ihr identisch ist): Im von Till Dembeck und Rolf Parr herausgegebenen Handbuch Literatur und Mehrsprachigkeit kommen die Begriffe ,Fiktionalität', ,Fiktivität' oder ,Fiktionstheorie' gar nicht erst vor.

Bedauerlich - oder aber, mit Blick auf zukünftige Forschungsarbeiten: gerade erfreulich - ist diese einstweilen fehlende Verbindung zwischen Fiktionstheorie und literarischer Mehrsprachigkeit deshalb, weil sich hier durchaus eine Reihe interessanter Forschungsfragen auftut. Die interpretatorische Vermittlung literarischer Mehrsprachigkeit mit spezifischen Erzählund Referenzstrukturen - also etwa eine Kopplung von Mehrsprachigkeit und Kontrafaktik - bildet dabei nur eine von zahlreichen Optionen. Zu diskutieren wäre beispielsweise, wie das erschwerte Verständnis - oder mitunter auch die (kalkulierte) Unverständlichkeit - mehrsprachiger Texte sich auf die Imagination fiktionaler Welten auswirkt. Welchen Einfluss nehmen generell unterschiedliche Sprachkompetenzen auf die Imagination fiktionaler Welten? 
Mit Blick auf die Gattungstheorie wäre ferner zu fragen, ob eine funktionale oder anderweitige Differenz besteht zwischen dem Einsatz literarischer Mehrsprachigkeit in fiktionalen Kunstformen wie dem Roman oder dem fiktionalen Film und dem Einsatz literarischer Mehrsprachigkeit in nicht (eindeutig) fiktionalen Kunstformen wie der Lyrik (vgl. Zipfel 2016).

Einen besonders reizvollen Forschungsgegenstand im Grenzbereich zwischen Fiktionstheorie und Mehrsprachigkeitsforschung bilden fiktive Sprachen wie Elbisch, Klingonisch oder $\mathrm{Na}^{\prime}{ }^{7}{ }^{7}$, die speziell für fiktionale Welten entworfen wurden, dabei aber mitunter linguistisch hochgradig ausdifferenziert sind und auch von Sprecherinnen und Sprechern in der realen Welt erlernt und verwendet werden. So wurden beispielsweise während der frühen Phase der Corona-Krise das Elbische und Klingonische genutzt, um an der staatlichen Zensur in China vorbeizukommunizieren, die fiktive Sprachen nicht auf dem Radar hatte (vgl. Deuber 2020): Gerade die partielle Unverständlichkeit dieser ursprünglich aus fiktionalen Welten stammenden Sprachen ermöglichte die Entfaltung subversiver politischer Potenziale in der realen Welt. Ausgehend von der Beobachtung, dass derartige Sprachen insbesondere im Genrebereich der Fantasy und Science-Fiction zum Einsatz kommen (vgl. Kremer 2020: 326), wäre aus fiktionstheoretischer Perspektive danach zu fragen, welche Korrespondenz zwischen der Fiktivität bestimmter Sprachen in Literatur und Film und der rationalen (Un-) Möglichkeit oder der (Un-)Vorstellbarkeit fiktionaler Welten besteht (vgl. Odendahl 2015). Im Anschluss an das oben erwähnte Thema der Unverständlichkeit mehrsprachiger Texte wäre darüber hinaus zu untersuchen, wie unterschiedliche Manifestationen sprachlicher Unverständlichkeit rezeptionsseitig erlebt und bewertet werden, welche Unterschiede also etwa gemacht werden zwischen fiktiven - und mithin unverständlichen - und natürlichen, dabei aber subjektiv unbekannten - also letztlich ebenfalls unverständlichen Sprachen? Ein reizvolles Mischphänomen bilden hier fiktive Sprachen, die zwar aus natürlichen Sprachen zusammengesetzt sind oder auf solche zurückgehen, in toto aber fiktiv sind, wie etwa die russisch-englische Hybridsprache Nadsat in Anthony Burgess' A Clockwork Orange oder das Neusprech in George Orwell's 1984 (das sich wiederum kontrafaktisch auf Aspekte der nationalsozialistischen und stalinistischen Sprachpolitik bezieht [vgl. Young 1991]).

\footnotetext{
In der Forschung werden solche Sprachen oft als ,fiktionale Sprachen ' bezeichnet. Plausibler wäre allerdings die Bezeichnung ,fiktive Sprachen', da hier ja die Erfundenheit der jeweiligen Sprache (Ontologie) und nicht eine bestimmte, institutionalisierte Form des Umgangs mit bestimmten Medien (Pragmatik) relevant ist. Unsinnig erscheint die Bezeichnung,fiktionale Sprachen' nicht zuletzt deshalb, weil derartige Sprachen ja mitunter durchaus in der wirklichen Welt gesprochen werden.
} 
Jenseits derartiger Spezialfragen soll an dieser Stelle vor allem deshalb für eine Fiktionstheorie literarischer Mehrsprachigkeit plädiert werden, weil eine derartige Forschungsperspektive zur Beantwortung einer der grundlegenden Fragen der literarischen Mehrsprachigkeitsforschung beitragen könnte: der Frage nämlich, worin denn nun eigentlich das Proprium literarischer Mehrsprachigkeit besteht. Als einer der - gar nicht sehr zahlreichen - Bereiche der Literaturwissenschaft, die sich systematisch mit der Frage beschäftigen, worin die Strukturen und Funktionspotenziale künstlerischer Diskurse und nur dieser bestehen (vgl. Bunia 2007: 12 f.), vermag die Fiktionstheorie den Blick zu schärfen für die Potenziale genuin literarischer Mehrsprachigkeit. Ein solcher Fokus auf die Spezifika literarischer Mehrsprachigkeit wäre dabei mit einer Adressierung moralischer, ethischer oder politischer Fragen durchaus vereinbar - vorausgesetzt eben, dass man unter „Politik der Literatur“ nicht einfach die Darstellung von Politik in der Literatur oder die „Politik der Schriftsteller“ versteht, sondern vielmehr die Art und Weise, wie „Literatur als Literatur Politik betreibt“" (Rancière 2011: 13).

\author{
Dr. Michael Navratil \\ navratil@uni-potsdam.de \\ Universität Potsdam \\ Institut für Germanistik \\ Professur Neuere deutsche Literatur/19.-21. Jahrhundert \\ Am Neuen Palais 10, Haus 5 \\ 14469 Potsdam \\ GERMANY
}

\title{
Bibliography
}

Aragon, L. 1931. Persecuté persécuteur. Paris: Editions surréalistes.

Ávila-Cabrera, J. J. 2013. Subtitling Multilingual Films: The Case of Inglourious Basterds. - Revista electrónica de lingüística aplicada, 12, 87-100.

Bunia, R. 2007. Faltungen: Fiktion, Erzählen, Medien. Berlin: Erich Schmidt.

Butter, M. 2015. American Basterds: The Deconstruction of World War II Myths in Steven Soderbergh's The Good German and Quentin Tarantino's Inglourious Basterds. - S. M. Herrmann et al., eds., Poetics of Politics. Textuality and Social Relevance in Contemporary American Literature and Culture. Heidelberg: Universitätsverlag Winter, 81-99.

Cavallero, J. J. 2011. Hollywood's Italian American filmmakers: Capra, Scorsese, Savoca, Coppola, and Tarantino. Urbana, Illinois, Chicago, Illinois, Springfield, Illinois: University of Illinois Press. 
NAVRATIL

Danneberg, L. 2009. Kontrafaktische Imaginationen in der Hermeneutik und in der Lehre des Testimoniums. - L. Danneberg, C. Spoerhase, D. Werle, eds., Begriffe, Metaphern und Imaginationen in Philosophie und Wissenschaftsgeschichte. Wiesbaden: Harrassowitz, 287-449.

Dath, D. 2009. Ein schöner Albtraum ist sich selbst genug. - Frankfurter Allgemeine Zeitung, 15.10.2008 - https://www.faz.net/aktuell/feuilleton/buecher/rezensionen/ belletristik/ein-schoener-albtraum-ist-sich-selbst-genug-1716257.html?printPage dArticle=true\#pageIndex_2 (28.04.2020).

Deuber, L. 2020. Chronologie einer Vertuschung. - Süddeutsche Zeitung, 03.04.2020. https://projekte.sueddeutsche.de/artikel/politik/coronavirus-in-wuhan-chronikder-vertuschung-e418140/?reduced $=$ true \&autologin=true (29.04.2020).

Evans, R. J. 2014. Veränderte Vergangenheiten: Über kontrafaktisches Erzählen in der Geschichte. München: Deutsche Verlags-Anstalt.

Kant, I. 1974 [1781]. Kritik der reinen Vernunft. Frankfurt a.M.: Suhrkamp.

Kracht, C. 2008. Ich werde hier sein im Sonnenschein und im Schatten. Köln: Kiepenheuer \& Witsch.

Kremer, C. 2020. Film. - T. Dembeck, R. Parr, eds., Literatur und Mehrsprachigkeit. Tübingen: Narr Francke Attempo, 321-328.

Lorenz, M. N. 2018. Christian Kracht liest Heart of Darkness: Zur Funktion einer intertextuellen Bezugnahme. - M. N. Lorenz, C. Riniker, eds., Christian Kracht revisited: Irritation und Rezeption. Berlin: Frank \& Timme, 421-453.

Navratil, M. 2019. Jenseits des politischen Realismus: Kontrafaktik als Verfahren politischen Schreibens in der Gegenwartsliteratur (Juli Zeh, Michel Houellebecq). - S. Neuhaus, I. Nover, eds., Das Politische in der Literatur der Gegenwart. Berlin, Boston: De Gruyter, 359-375. https://doi.org/10.1515/9783110568561020

Navratil, M. (im Druck). Lying in Counterfactual Fiction. On the Critical Function of Metafactuality. - M. Fludernik, S. Packard, eds., Being Untruthful: Lies, Fictionality and Related Nonfactualities. Baden-Baden: Ergon.

Navratil, M. (in Vorbereitung). Kontrafaktik der Gegenwart. Politisches Schreiben als Realitätsvariation bei Christian Kracht, Kathrin Röggla, Juli Zeh und Leif Randt. Berlin, Boston: De Gruyter.

Neitzel, S. 2003. Was wäre wenn...? Gedanken zur kontrafaktischen Geschichtsschreibung. - T. Stamm-Kuhlmann et al., eds., Geschichtsbilder. Festschrift für Michael Salewski zum 65. Geburtstag. Stuttgart: Franz Steiner, 312-324.

Nover, I. 2012. Referenzbegehren: Sprache und Gewalt bei Bret Easton Ellis und Christian Kracht. Köln, Weimar, Wien: Böhlau.

Odendahl, J. 2015. Die Kunst des Möglichen: Über den Wirklichkeitsbezug phantastischer Literatur. - Wirkendes Wort, 65 (2), 261-279.

Radaelli, G. 2011. Literarische Mehrsprachigkeit: Sprachwechsel bei Elias Canetti und Ingeborg Bachmann. Berlin: Akademie Verlag.

Rancière, J. 2011. Politik der Literatur. 2., überarbeitete Aufl. Wien: Passagen-Verlag.

Rosenfeld, G. D. 2005. The World Hitler Never Made. Alternate History and the Memory of Nazism. Cambridge et al.: Cambridge University Press. 
Sprach- und Weltalternativen

Schmitz-Emans, M., ed., 2004. Literatur und Vielsprachigkeit. Heidelberg: Synchron Wissenschaftsverlag der Autoren.

Schöll, J. 2014. Die Schweizer Matrix: Intertextuelle und intermediale Konstruktionen der Nation in Christian Krachts Roman Ich werde hier sein im Sonnenschein und im Schatten. - J. Preece, ed., Re-forming the Nation in Literature and Film: The Patriotic Idea in Contemporary German-Language Culture. Oxford et al.: Lang, 289-307.

Sepp, A. 2020. Ethik der Mehrsprachigkeit. - T. Dembeck, R. Parr, eds., Literatur und Mehrsprachigkeit. Tübingen: Narr Francke Attempo, 53-65.

Singles, K. 2013. Alternate History. Playing with Contingency and Necessity. Berlin, Boston: De Gruyter.

Tarantino, Q. 2009. Inglourious Basterds. A Screenplay. New York: Weinstein Books, Little, Brown and Company.

Tarantino, Q. (Regie) 2009. Inglourious Basterds. USA, Germany.

Young, J. W. 1991. Totalitarian Language: Orwell's Newspeak and its Nazi and Communist Antecedents. Charlottesville et al.: University Press of Virginia.

Zipfel, F. 2016. Lyrik und Fiktion. - D. Lamping, ed., Handbuch Lyrik. Theorie, Analyse, Geschichte. 2., erweiterte Aufl. Stuttgart: J. B. Metzler, 184-188.

Zymner, R. 2020. Erzählen. - T. Dembeck, R. Parr, eds., Literatur und Mehrsprachigkeit. Tübingen: Narr Francke Attempo, 293-297. 